\title{
PTEN sensitizes MDA-MB-468 cells to inhibition of MEK/Erk signaling for the blockade of cell proliferation
}

\author{
KIBEOM JANG, MINSOON KIM, HYE-SOOK SEO and INCHEOL SHIN \\ Department of Life Science, College of Natural Sciences, Hanyang University, Seoul 133-791, Korea
}

Received April 9, 2010; Accepted June 1, 2010

DOI: 10.3892/or_00000922

\begin{abstract}
Phosphatase and tensin homolog (PTEN) is a tumor suppressor that inhibits PI3K/Akt signaling. To examine the effect of PTEN on breast cancer cell proliferation, we expressed PTEN in MDA-MB-468 cells (MDA-MB-468 PTEN) by retroviral infection and tested the cell proliferation rate. We found that the growth rate of MDA-MB-468 PTEN cells was significantly lower than that of MDA-MB-468 control vector cells (MDA-MB-468 vec). When the PI3K/Akt signaling inhibitor LY294002 and the MEK/Erk signaling inhibitor U0126 were applied, LY294002 reduced cell proliferation in MDA-MB-468 PTEN and MDA-MB-468 vec by $20 \%$, while U0126 led to a $>60 \%$ reduction in MDA-MB-468 PTEN and a $20 \%$ reduction in MDA-MB-468 vec cells. FACS analysis demonstrated that the subG0/G1 apoptotic fraction was significantly increased in MDA-MB-468 PTEN cells after U0126 treatment, while LY294002 treatment in both cell lines and U0126 treatment in MDA-MB-468 vec cells led to a modest increase in the apoptotic fraction. This phenomenon was accompanied by the down-regulation of p-Erk. p-Erk levels were significantly lower after U0126 treatment in MDA-MB-468 PTEN cells. Similar results were observed in MDA-MB-231 cells, which express endogenous PTEN. The growth of MDA-MB-231 cells was significantly inhibited after U0126 treatment, compared to LY294002, while PTEN-null ZR-75-1 cells did not show increased sensitivity to U0126 over LY294002. Taken together, these findings suggest that blockade of PI3K/Akt signaling by PTEN may render breast cancer cells more dependent on the MEK/Erk pathway for their proliferation and survival.
\end{abstract}

\section{Introduction}

The phosphatidylinositol 3-kinase (PI3K) and Ras/Raf signaling pathways are mutated in many human cancers

Correspondence to: Dr Incheol Shin or Dr Hye-Sook Seo, Department of Life Science, College of Natural Sciences, Hanyang University, Seoul 133-791, Korea

E-mail: incheol@hanyang.ac.kr

E-mail: phyto-seo@hotmail.com

Key words: breast cancer, PTEN, Akt, MEK/Erk
(1). Activated PI3K induces the conversion of the plasma membrane lipid phosphatidylinositol $(4,5)$-bisphosphate $\left(\mathrm{PIP}_{2}\right)$ to phosphatidylinositol $(3,4,5)$-triphosphate $\left(\mathrm{PIP}_{3}\right)$ by phosphorylation at the D-3 position of the inositol ring (2). A 3 -phosphoinositide-dependent protein kinase, PDK1, is then activated by $\mathrm{PIP}_{3}$, resulting in phosphorylation of the activation loop of Akt at Thr308 (3). Upstream signaling can also activate mammalian target of rapamycin (mTOR) complex 2 (mTORC2) and mTORC2 phosphorylates the hydrophobic motif of Akt at Ser473 to fully activate Akt $(4,5)$. Activated Akt plays very important roles in cell survival, cell growth, cell proliferation, angiogenesis and cellular metabolism (5). Phosphatase and tensin homolog (PTEN) down-regulates Akt activation by dephosphorylation of $\mathrm{PIP}_{3}$ at the D-3 position of the inositol ring (6).

Mitogen-activated protein kinases (MAPKs) are a family of Ser/Thr protein kinases that are involved in many cellular functions, such as proliferation, differentiation, inflammation, cell movement and cell death (7). MAPKs are phosphorylated and activated by MAPK-kinases (MAPKKs) and phosphorylate various targets, including transcription factors and MAPK-activated protein kinases (MAPKAPKs). MAPKs are dephosphorylated and inactivated by a family of MAPKphosphatases (MKPs). MAPKKs are phosphorylated and activated by MAPKK-kinases (MAPKKKs). The MAPKKKs are then activated by interaction with a family of small GTPases and/or other protein kinases, connecting the MAPK module to cell surface receptors or external stimuli (growth factors, mitogens, stress) (8). There are six distinct groups within the MAPK family in mammals, including extracellular signal-regulated kinases 1 and 2 (Erk1/2), c-Jun-N-terminal kinases (JNKs), p38, Erk5, Erk3/4 and Erk7/8 (8-10).

The PTEN gene, a novel tumor suppressor, is localized to chromosome 10q23.3 and shares extensive homology with the cytoskeletal protein, tensin (11). A high frequency of mutations at the PTEN locus has been described in cancers of the brain, breast and prostate (12). Reportedly, PTEN not only antagonizes tumorigenesis, but also sensitizes cancer cells to chemotherapy and decreases drug resistance (13-15).

Based on the key roles of PTEN and PI3K/Akt in cancer, we investigated the potential therapeutic role of PTEN in breast cancer. We reconstructed PTEN in PTEN-null MDAMB-468 breast cancer cells (MDA-MB-468 PTEN) by retroviral infection and compared the proliferation rate and phosphorylation status of signaling effector molecules between MDA-MB-468 PTEN cells and MDA-MB-468 control vector 
(MDA-MB-468 vec) cells. We also investigated the effects of the PI3K/Akt inhibitor LY294002 and the MEK/Erk inhibitor U0126 on cell proliferation in MDA-MB-468 PTEN and MDA-MB-468 vec cells.

\section{Materials and methods}

Cell culture. MDA-MB-468 vec and MDA-MB-468 PTEN cells were developed as described by Bianco et al (16) and were cultured in DMEM/F12 (Gibco, Carlsbad, CA, USA) (1:1). MDA-MB-231, ZR-75-1 and 293T cells were cultured in DMEM (Gibco). All media contained 10\% FBS, $100 \mathrm{U} / \mathrm{ml}$ penicillin and $100 \mathrm{mg} / \mathrm{ml}$ streptomycin (Welgene, Daegu, Korea). All cell lines were incubated at $37^{\circ} \mathrm{C}$ with $5 \% \mathrm{CO}_{2}$ in a humidified atmosphere.

Antibodies and reagents. Akt, p-Akt (Ser473), c-Raf, p-c-Raf, MEK, p-MEK and $B$-actin antibodies were purchased from Cell Signaling Technology (Beverly, MA, USA). PTEN, Erk and p-Erk antibodies were purchased from Santa Cruz Biotechnology (Santa Cruz, CA, USA). LY294002 and U0126 were obtained from Calbiochem (Gibbstown, NJ, USA).

Proliferation assays. Cells were seeded at a density of $5 \times 10^{4}$ cells/well in a 12 -well dish. Cells were trypsinized and counted in triplicate using a hemocytometer for 5 days.

Western blotting and immunoprecipitation. Cells were washed once with phosphate-buffered saline (PBS) and lysed in a lysis buffer [20 mM Tris- $\mathrm{HCl}, \mathrm{pH} 7.4,0.1 \mathrm{mM}$ EDTA, $150 \mathrm{mM} \mathrm{NaCl}, 1 \% \mathrm{NP}-40,0.1 \%$ Triton $\mathrm{X}-100,0.1 \%$ SDS, $20 \mathrm{mM} \mathrm{NaF}, 1 \mathrm{mM} \mathrm{Na} \mathrm{VO}_{4}$ and $1 \mathrm{X}$ protease inhibitor (Roche)]. All protein samples were resolved using 10\% SDSPAGE after boiling for $10 \mathrm{~min}$ in SDS sample buffer. For Western blot analysis, proteins were electro-transferred onto nitrocellulose membranes over the course of $2 \mathrm{~h}$. The membranes were blocked with 5\% skim milk in TBST for $1 \mathrm{~h}$ and incubated with appropriated dilutions of primary antibody in TBST containing $5 \%$ bovine serum albumin (BSA) overnight. The membranes were then washed three times with TBST and incubated with secondary antibody solution for $2 \mathrm{~h}$. Blots were visualized with the WESTZOL-plus detection system (iNtRON Biotechnology, Seoul, Korea).

Cell cycle analyses by flow cytometry. Cells were harvested with $0.25 \%$ trypsin and washed once with PBS. After centrifugation, cells were fixed in $100 \%$ ice-cold methanol overnight at $-20^{\circ} \mathrm{C}$. Cells were incubated in $50 \mu \mathrm{g} / \mathrm{ml}$ propidiumiodide (PI) in PBS and $1 \mathrm{mg} / \mathrm{ml}$ RNAase in PBS for 30 min. Cell cycle analysis was performed using FACS calibur (Becton-Dickinson Biosciences, Mountain View, CA, USA), and the data was analyzed using Cell Quest software. Each experiment was repeated three times.

\section{Results}

PTEN expression down-regulates the proliferation rate of $M D A-M B-468$ cells. We first compared the proliferation rate between MDA-MB-468 PTEN cells (which permanently
A

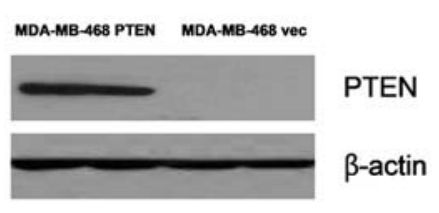

B

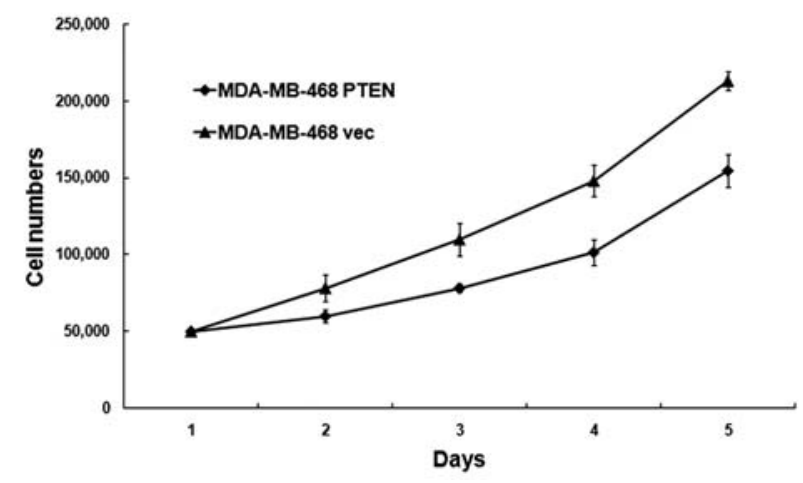

Figure 1. (A) Retroviral expression of PTEN in MDA-MB-468 breast cancer cells. MDA-MB-468 cells were infected with retroviruses encoding PTEN or p.BMN vector (vec). (B) Activation of PTEN down-regulates the growth of MDA-MB-468 cells. Cells were seeded at a density of $5 \times 10^{4}$ cells/well in a 12-well dish and counted with a hemocytometer for 5 days.

express PTEN by retroviral infection) and MDA-MB-468 vec (vector control) cells. As seen in Fig. 1, we found that the growth rate of MDA-MB-468 PTEN was lower than that of the MDA-MB-468 vec cells $\left(1.55 \times 10^{5}\right.$ cells $/ 2.13 \times 10^{5}$ cells at 5 days after the cell seeding), suggesting that forced expression of PTEN inhibits the proliferation of MDA-MB-468. This is possibly due to blockade of PI3K/Akt activity.

The effect of signaling inhibitors LY294002 and U0126 on the proliferation rate of $M D A-M B-468$ cells. To investigate the effect of PI3K/Akt and MEK/Erk signaling inhibitors on the cell proliferation rate of MDA-MB-468 cells, we seeded the cells at a density of $5 \times 10^{4}$ cells/well in a 12-well dish, treated the cells with DMSO (vehicle), LY294002 $(10 \mu \mathrm{M})$, or U0126 $(10 \mu \mathrm{M})$ every $48 \mathrm{~h}$, and counted the cells every day using a hemocytometer (Fig. 2A). We found that treatment with LY294002 resulted in a roughly $20 \%$ reduction in cell proliferation after $120 \mathrm{~h}$ in both cell lines. Meanwhile, U0126 treatment led to a $>60 \%$ reduction in MDA-MB-468 PTEN cells, compared to a $20 \%$ reduction in MDA-MB-468 vec cells (Fig. 2B). These results indicate that expression of PTEN may confer sensitivity to a MEK/Erk inhibitor, resulting in the blockade of cell proliferation in MDA-MB468 cells. Our results show that synergy between PTEN and inhibition of MEK/Erk strongly blocks cell proliferation in MDA-MB-468 cells.

The effects of LY294002 and U0126 on the phosphorylation of intracellular effector molecules. To examine the status of intracellular signaling molecules in MDA-MB-468 cells following treatment with LY294002 or U0126, we seeded the cells at a density of $1 \times 10^{6}$ cells in $60 \mathrm{~mm}$ dishes and treated the cells with DMSO (vehicle), LY294002 (10 $\mu \mathrm{M})$ or U0126 $(10 \mu \mathrm{M})$. After either $30 \mathrm{~min}$ or $24 \mathrm{~h}$ of treatment, we prepared protein extracts and performed Western blot analysis. As 
A

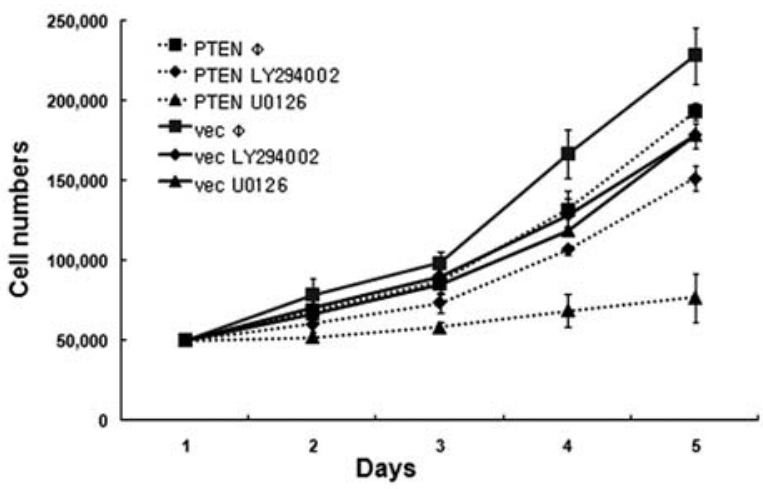

B

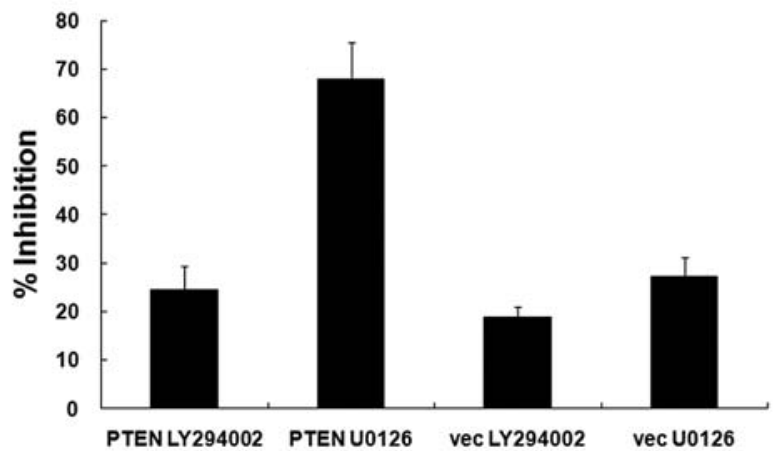

Figure 2. (A) Proliferation of MDA-MB-468 vec and MDA-MB-468 PTEN cells after treatment with PI3K/Akt and MEK/ERK inhibitors. Cells were seeded at a density of $5 \times 10^{4}$ cells/well in a 12-well dish and counted with a hemocytometer for 5 days. Both LY294002 and U0126 were used at concentrations of $10 \mu \mathrm{M}$. Medium (containing fresh inhibitors) was replaced every 2 days. (B) Inhibition of cell proliferation of MDA-MB-468 vec and MDA-MB-468 PTEN following treatment with PI3K/Akt and MEK/ERK inhibitors. Inhibition rate of cell proliferation of MDA-MB-468 vec and MDA-MB-468 PTEN treated with LY294002 and U0126 was measured by proliferation assay. Cells were treated with inhibitor (at a concentration of $10 \mu \mathrm{M}$ ) for 5 days. Medium (containing fresh inhibitors) was replaced every 2 days. expected, we did not find PTEN expression in MDA-MB$468 \mathrm{vec}$ cells, but found strong p-Akt activity as a result of the loss of functional PTEN (Fig. 3). When the PI3K/Akt inhibitor LY294002 was added, down-regulation of p-Akt occurred in MDA-MB-468 vec cells. Interestingly, when the MEK/Erk inhibitor U0126 was added, the level of p-Erk did not change in MDA-MB-468 vec cells, but was dramatically lower in MDA-MB-468 PTEN cells as early as 30 min after treatment (Fig. 3). This suggests that PTEN expression confers sensitivity to the MEK/Erk inhibitor in the down-regulation of p-Erk in MDA-MB-468 cells. In our study, Erk activation was significantly decreased only when both PI3K/Akt signaling and MEK/Erk were blocked by PTEN expression and U0126 treatment, respectively. On the other hand, Erk was constantly activated in MDA-468 vec cells, even in the presence of either LY294002 or U0126, as well as in MDA-468 PTEN cells in the presence of LY294002. Interestingly, phosphorylation of c-Raf and MEK (upstream kinases of Erk) was significantly lower in MDA-MB-468 PTEN cells, as compared with MDAMB-468 vec cells (Fig. 4). Overexpression of PTEN decreased phosphorylation of c-Raf both at Ser338 [which is required for Raf activation (17)] and Ser259 [an Akt consensus inhibitory phosphorylation site in a regulatory domain of c-Raf (18)]. This finding suggests that Erk may be activated by a Raf/MEK-independent pathway in MDA-MB-468 PTEN cells.

Cell cycle analysis of MDA-MB-468 PTEN and MDA-MB468 vec following treatment with LY294002 and U0126. For cell cycle analysis, MDA-MB-468 PTEN and MDA-MB-468 vec cells were treated with LY294002 and U0126 at a concentration of $10 \mu \mathrm{M}$ for $48 \mathrm{~h}$, and propodium iodide-labeled nuclei were analyzed by flow cytometry. We found that the subG0/G1 apoptotic fraction was significantly higher in MDAMB-468 PTEN cells after U0126 treatment for 48 h, while
A
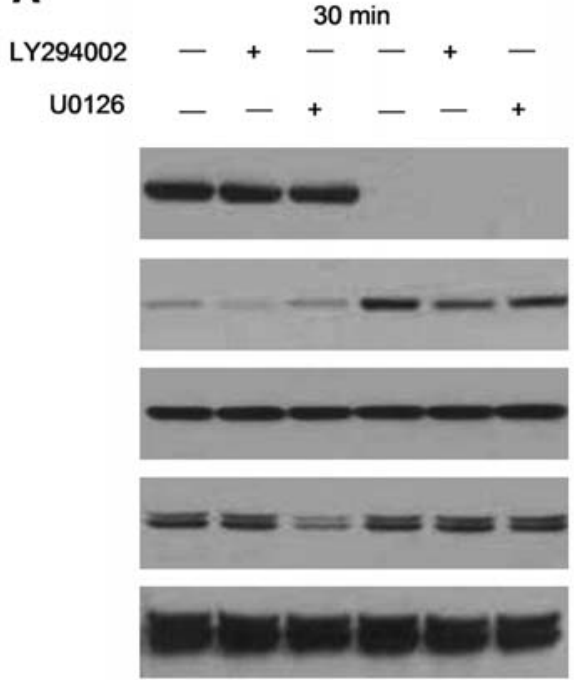

MDA-MB-468 PTEN MDA-MB-468 vec
B

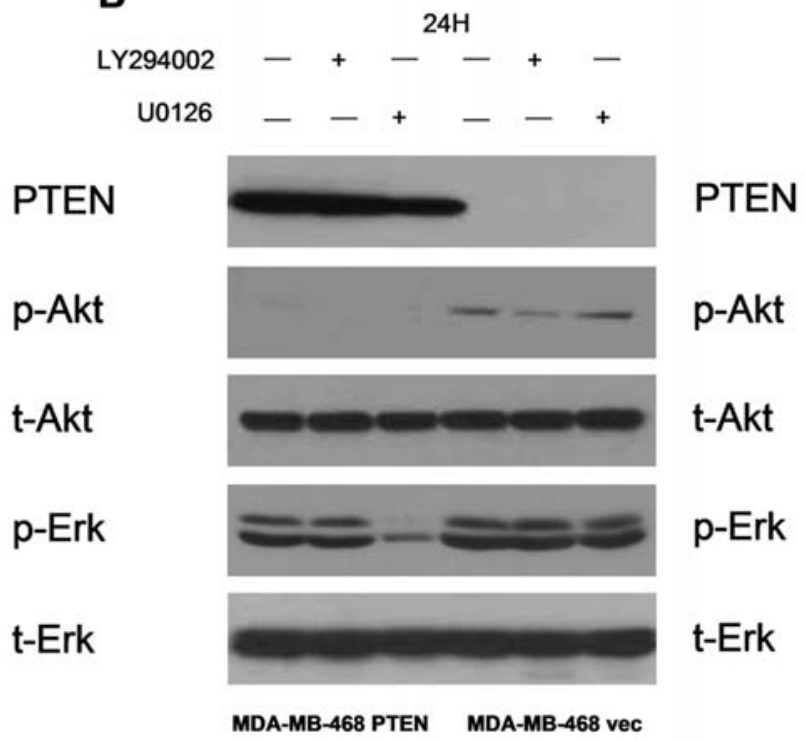

Figure 3. Effects of PI3K/Akt and MEK/ERK inhibitors on Akt and ERK phosphorylation. Cells were seeded at a density of $1 \times 10^{6}$ cells/dish in 60 mm dishes. The cells were then treated with LY294002 $(10 \mu \mathrm{M})$ or U0126 (10 $\mu \mathrm{M})$ for either $30 \mathrm{~min}(\mathrm{~A})$ or $24 \mathrm{~h}(\mathrm{~B})$. After treatment, the cells were subjected to Western blot analyses with each of the indicated antibodies. 
A

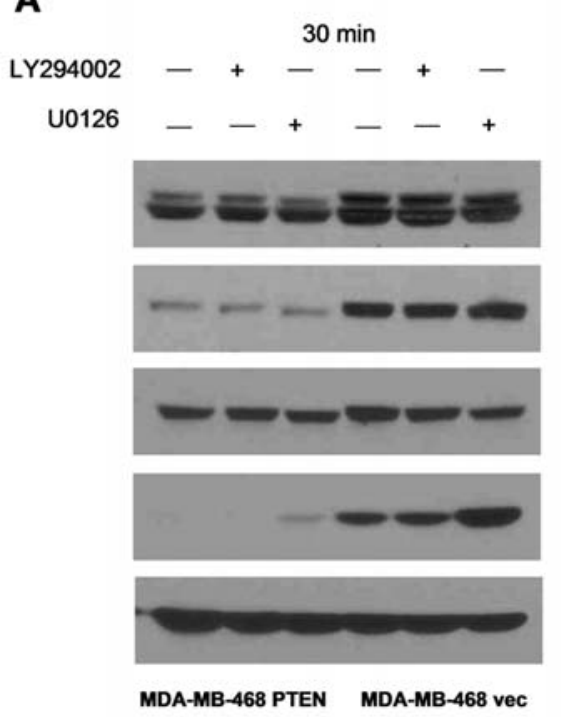

B

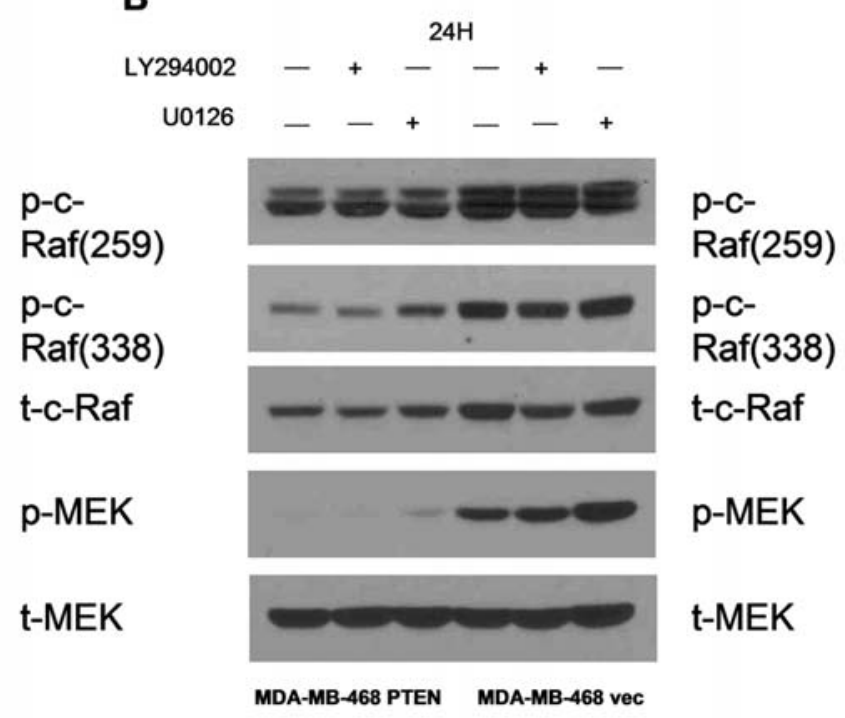

Figure 4. Effects of PI3K/Akt and MEK/ERK inhibitors on c-Raf and MEK phosphorylation. Cells were seeded at a density of $1 \times 10^{6}$ cells/dish in 60 mm dishes. The cells were then treated with LY294002 $(10 \mu \mathrm{M})$ or U0126 $(10 \mu \mathrm{M})$ for either $30 \mathrm{~min}(\mathrm{~A})$ or $24 \mathrm{~h}(\mathrm{~B})$. After treatment, the cells were subjected to Western blot analyses with each of the indicated antibodies.

A
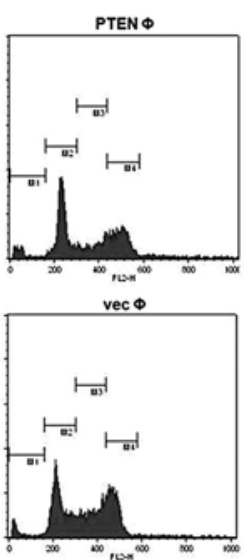

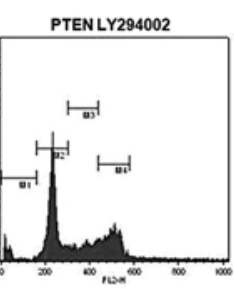

vec LY294002

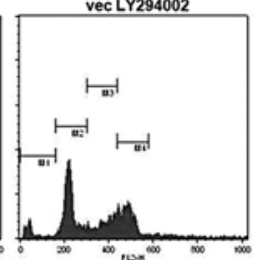

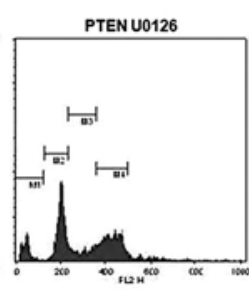

vec 00126

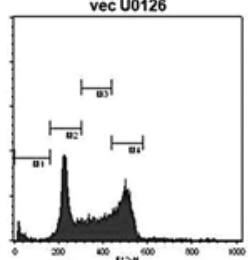

B

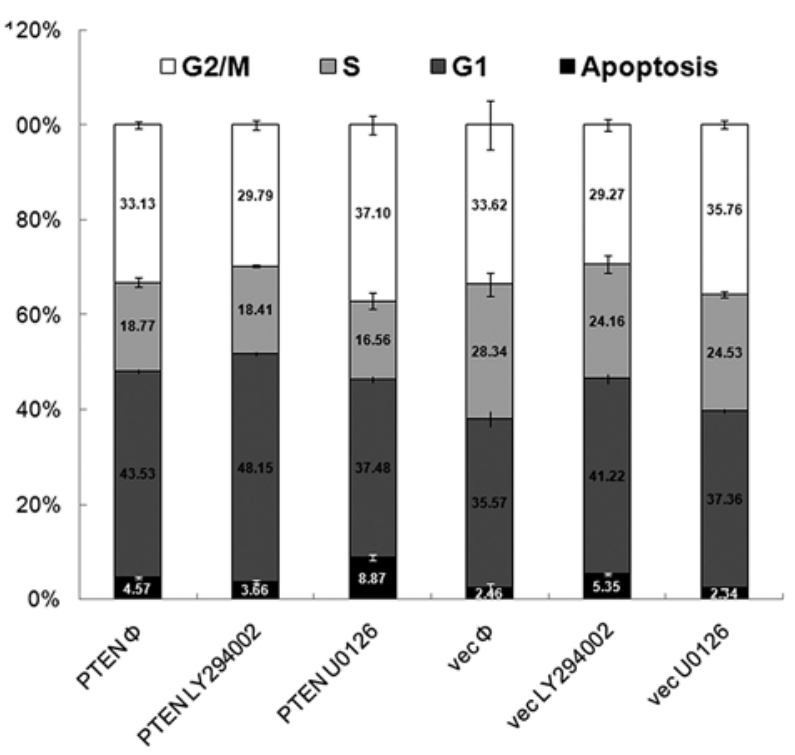

Figure 5. (A) Cell cycle analysis of MDA-MB-468 vec and MDA-MB-468 PTEN upon treatment with LY294002 and U0126. Cells were seeded at a density of $1 \times 10^{6}$ cells/dish in $100 \mathrm{~mm}$ dishes. Cells were treated with LY294002 or U0126 (10 $\mu \mathrm{M}$ each) for $48 \mathrm{~h}$. After treatment, the cells were fixed in methanol and incubated in PBS containing $50 \mu \mathrm{g} / \mathrm{ml}$ propodium iodide and $1 \mathrm{mg} / \mathrm{ml}$ RNase. Propodium iodide-labeled nuclei were analyzed by flow cytometry. (B) DNA histograms in (A) represented as bar graphs. Each experiment was performed 3 times, with the mean values and standard deviations indicated.

LY294002 treatment in both cell lines and U0126 treatment in MDA-MB-468 vec cells led to a modest increase in the apoptotic fraction (Fig. 5). This indicates that the decrease in proliferation rate of MDA-MB-468 PTEN cells following U0126 treatment may be due to an increase in apoptotic cell death in these cells. Moreover, we found a proteolytic cleavage form of caspase-3 (an apoptosis marker) only following treatment with U0126 in MDA-MB-468 PTEN cells (Fig. 6). This suggests that the synergy between the inhibition of PI3K/Akt by PTEN and the blockade of MEK/Erk by U0126 induces apoptosis, resulting in significant inhibition of cell growth in MDA-MB-468 PTEN cells.

The effect of LY294002 and U0126 on cell proliferation rate in MDA-MB-231 and ZR-75-1 cells. To investigate whether endogenous PTEN exerts the same effect as reconstructed PTEN in MDA-MB-468 cells, we compared the proliferation rate between MDA-MB-231 cells and ZR-75-1 cells. MDAMB-231 cells express endogenous PTEN, while ZR-75-1 cells contain a hemizygous deletion of PTEN and a missense 


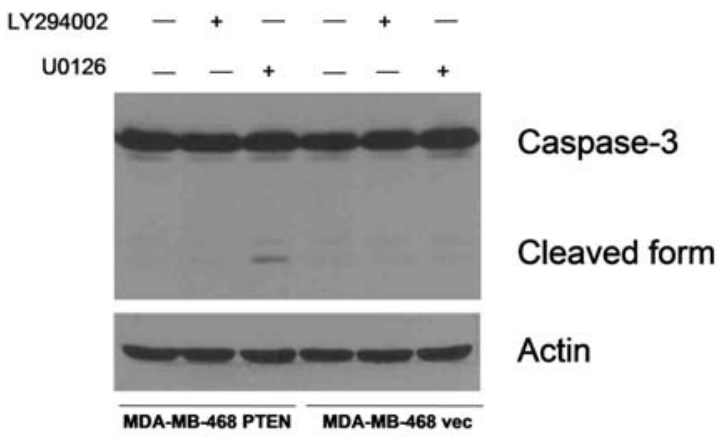

Figure 6. Effects of PI3K/Akt and MEK/ERK inhibitors on the cleavage of caspase-3. Cells were seeded at a density of $1 \times 10^{6}$ cells/dish in $100 \mathrm{~mm}$ dishes. The cells were then treated with LY294002 $(10 \mu \mathrm{M})$ or U0126 $(10 \mu \mathrm{M})$ for $48 \mathrm{~h}$. After treatment, the cells were subjected to Western blot analyses with caspase- 3 antibodies.

mutation in the other allele (19) and express very low levels of PTEN (Fig. 7A). As expected, p-Akt activity was lower in MDA-MB-231 cells than in ZR-75-1 cells (Fig. 7A). In accordance with the previous results with MDA-MB-468 cells, treatment with LY294002 resulted in a $25 \%$ reduction in cell proliferation after $96 \mathrm{~h}$ in both cell lines, while U0126 treatment led to a $>45 \%$ reduction in MDA-MB-231 cells (Fig. 7B) and a 25\% reduction in ZR-75-1 cells (Fig. 7C). This indicates that endogenous PTEN also confers sensitivity to $\mathrm{MEK} /$ Erk inhibition for the blockade of cell proliferation in breast cancer cells. Moreover, we also found that U0126 treatment inhibited the activation of Erk (p-Erk) in MDA-
MB-231 cells (Fig. 7A), suggesting that natural PTEN and U0126 treatment combine to block the phosphorylation of Erk and inhibit cell growth.

\section{Discussion}

In this study, we investigated the role of PTEN as a sensitizer for MEK/Erk inhibition, which then results in a decrease in cell proliferation and a reduction in p-Erk activity in MDAMB-468 breast cancer cells. MDA-MB-468 cells lack PTEN expression and function due to a frameshift mutation in one PTEN allele and loss of the other PTEN allele (20). Accordingly, we used this cell line for our study and expressed PTEN through retroviral infection. Our data indicate that MDA-MB-468 PTEN cells (which artificially express PTEN) grew slowly, compared to MDA-MB-468 vec cells, suggesting that PTEN acts as a growth suppressor. Interestingly, the presence of PTEN generated a greater sensitivity to the MEK/ Erk inhibitor U0126, compared to the PI3K/Akt inhibitor, LY294002. On the other hand, the growth of MDA-MB-468 vec cells, which do not express PTEN, was only slightly inhibited by LY294002 and U0126; this suggests that PTEN sensitizes breast cancer cells to the MEK/Erk inhibitor for the blockade of cell proliferation (Fig. 2). Notably, this strong growth inhibition of MDA-MB-468 PTEN cells following U0126 treatment was followed by an increase in apoptosis (Fig. 5).

The MEK/Erk pathway appears to engage in cross-talk with the PI3K/Akt/PTEN signaling pathway. It has been reported that PTEN inhibits the integrin- and growth factor-
A



B

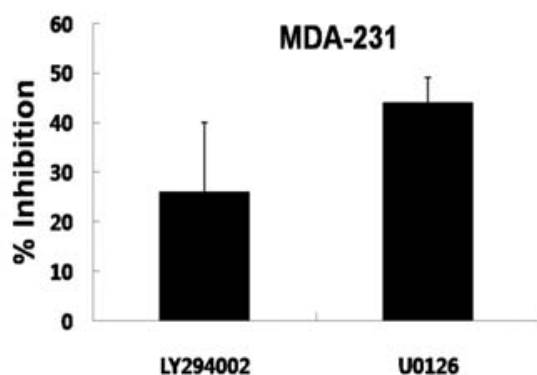

C

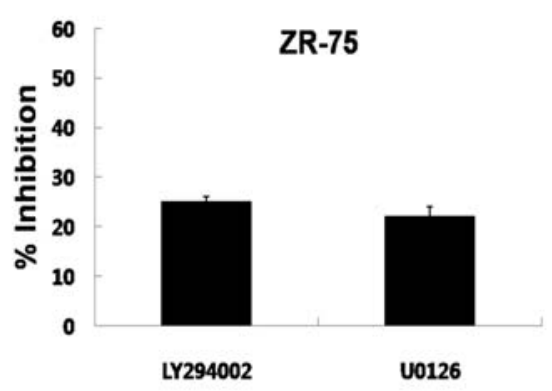

Figure 7. (A), (B) Effect of LY294002 and U0126 on cell proliferation rate of MDA-MB-231 and ZR-75-1 cells. MDA-MB-231 cells (A) and ZR-75-1 (B) cells were seeded at a density of $2 \times 10^{4}$ cells/well in a 12-well dish and counted with a hemocytometer for 5 days. Treatment with LY294002 and U0126 was at a concentration of $10 \mu \mathrm{M}$. Medium (containing fresh inhibitors) was replaced every 2 days. (C) Effects of PI3K/Akt and MEK/ERK inhibitors on Akt and ERK phosphorylation in MDA-MB-231 and ZR-75-1 cells. MDA-MB-231 and ZR-75-1 cells were seeded at a density of 1x10 ${ }^{6}$ cells/dish in 60 mm dishes. The cells were then treated with LY294002 $(10 \mu \mathrm{M})$ or U0126 $(10 \mu \mathrm{M})$. After treatment, the cells were subjected to Western blot analyses with each of the indicated antibodies. 
mediated Erk signaling pathways (21). It has also been observed that there is cross-talk between the PI3K/Akt and MEK/Erk pathways in cell growth: inhibition of PI3K or MEK slowed the differentiation of immature MoDCs and induced apoptosis in 10-30\% of cultured cells, while inhibition of both PI3K and MEK resulted in apoptosis in $70 \%$ of the cells (22). Similarly, our data confirmed that there is crosstalk between PTEN and inhibition of the MEK/Erk signaling pathway, as the combination induces stronger cell growth inhibition and weaker p-Erk activity.

Erk is phosphorylated and activated by Ras/Raf/MEK signaling, and p-Erk regulates cell proliferation and differentiation $(23,24)$. However, it should be noted that Erk is also activated by Raf- or MEK-independent pathways. In this study, we observed that PTEN suppressed the expression of both p-Raf and p-MEK while failing to decrease p-Erk activity in MDA-MB-468 cells (Figs. 3 and 4). Erk was constantly activated in MDA-MB-468 vec cells in all conditions and in MDA-MB-468 PTEN cells in the presence or absence of LY294002. The inhibition of Erk activation occurred only in MDA-MB-468 PTEN cells treated with U0126, suggesting that only the combined inhibition of PI3K and MEK was able to down-regulate Erk activity. In the presence of high PI3K activity due to PTEN mutation, p-Erk appears to be regenerated via Raf/MEK-independent pathways in MDA-MB-468 cells. Interestingly, overexpression of PTEN down-regulated the phosphorylation of c-Raf at both the inhibitory (Ser259) and activating (Ser338) sites (Fig. 4). The inhibition of phosphorylation of c-Raf at Ser259 (the Akt consensus site) maybe not directly mediated by the inhibition of Akt activity by PTEN in MDA-MB-231 PTEN cells because inhibition of p-Akt by LY294002 did not efficiently block c-Raf phosphorylation at Ser259 in MDA-MB-231 vec cells (Fig. 4). Indeed, previous reports indicate that the inhibition of Raf/MEK/Erk by Akt may be not the universal mechanism. Laprise et al (25) reported that PI3K/Akt inhibition results in activation of the Raf/MEK/Erk pathway in differentiating enterocytes, but not in proliferative cells.

It has been reported that PKC $\alpha$ may activate MEK independent of Raf and Ras to trigger sustained Erk signaling and cell cycle arrest of HepG2 induced by TPA (26). It was also reported that Epigallocatechin-3-gallate inhibits PC-3 prostate cancer cell proliferation via MEK-independent Erk1/2 activation (27). Moreover, there is evidence of MEK-independent pathways that regulate the prolonged activation of Erk by platelet-derived growth factor signaling (28). In agreement with these previous reports, our data indicate that Erk can be activated in Raf/MEK-independent manner in MDA-MB-468 cells. The inhibitory effect of ectopic PTEN on the phosphorylation of c-Raf and c-MEK may be mediated by direct dephosphorylation of c-Raf and MEK by exogenous PTEN protein phosphatase activity in MDA-MB-468 PTEN cells, or by the perturbation of other signaling pathways responsible for Raf/MEK phosphorylation by expression of PTEN. Indeed, it has been reported that the protein phosphatase activity of PTEN blocks Sp1 phosphorylation by inactivation of Erk, MEK and PKC, which is eventually involved in the downregulation of IGF-II expression in hepatoma cell lines (29).

PI3K/Akt signaling and MEK/Erk signaling are the main processes involved in the induction of proliferation in many types of cells. Because of the blockade of functional PI3K/ Akt signaling by PTEN expression, MDA-MB-468 PTEN cells depend more on the MEK/Erk pathway (i.e., addiction to MEK/Erk signaling). As such, the treatment of MDA-468PTEN cells with U0126, which blocks the MEK/Erk pathway, contributes to stronger suppression of cell growth and induction of apoptotic cell death as a result of blocking both the PI3K/Akt and MEK/Erk signaling pathways. In line with our data, it was recently reported that loss of PTEN markedly decreased the response to MEK inhibition in basal-like cancer cells (including MDA-MB-468 cells), and that combined inhibition of both PI3K and MEK synergistically inhibited the growth of basal-like breast cancer cells both in vitro and in vivo (30). Basal-like breast cancers are a subtype of breast cancer receiving this name due to the resemblance of their gene expression profile to that of the normal breast epithelial cells (31). Absence of both functional nuclear hormone receptors and HER2 gene amplification are characteristics of basal-like breast cancers; hence, this subtype of the disease is often called 'triple-negative' breast cancer (32).

Fig. 7 indicates that MDA-MB-231 cells expressing endogenous PTEN were more sensitive to MEK/Erk inhibition by U0126 than ZR-75-1 cells, which express very low levels of endogenous PTEN. Of note, the percentage inhibition of cell growth is shown in Fig. 7, as there is a big difference in growth rates between MDA-MB-231 and ZR-75-1, making it difficult to compare the two directly. Again, p-Erk levels in ZR-75-1 cells were not affected by U0126, while p-Erk levels in MDA-MB-231 cells were significantly reduced by U0126. These data confirm that both endogenous and exogenous PTEN are capable of conferring sensitivity to U0126. Indeed, among basal-like breast cancer cells, PTEN-null cells (such as MDA-MB-468, HCC1395, BT-549, CAL-148, HCC1937, HCC38, and MDA-MB-436) have a higher EC50 concentration for MEK inhibitor, compared to PTEN wild-type cells (including MDA-MB-144VI, HDQ-P1, DU4475, HCC1806, MDA-MB-231, HCC1143 and BT20) (30).

In conclusion, our study demonstrates that the presence of PTEN confers sensitivity to MEK/Erk inhibition for the blockade of cell proliferation and down-regulation of p-Erk levels in basal-like breast cancer MDA-MB-468 cells. Combination treatment with PI3K/Akt inhibitors and MEK/ Erk inhibitors could be a potentially effective therapeutic strategy for basal-like triple-negative breast cancers without a target molecule, such as estrogen receptor or HER2, for a tailored therapeutic approach.

\section{Acknowledgements}

This study was supported by grants A084466 from the Korea Healthcare Technology R\&D Project, Ministry of Welfare and Family Affairs, FPR09A2-080 of the 21st Century Frontier Functional Proteomics Projects from the Korean Ministry of Science and Technology, and 2009-0074945 from the Korea Research Foundation.

\section{References}

1. Shaw RJ and Cantley LC: Ras, PI(3)K and mTOR signalling controls tumour cell growth. Nature 441: 424-430, 2006.

2. Cantley LC: The phosphoinositide 3-kinase pathway. Science 296: 1655-1657, 2002. 
3. Brazil DP and Hemmings BA: Ten years of protein kinase B signalling: a hard akt to follow. Trends Biochem Sci 26: 657-664, 2001.

4. Sarbassov DD, Guertin DA, Ali SM and Sabatini DM: Phosphorylation and regulation of $\mathrm{Akt} / \mathrm{PKB}$ by the rictor-mTOR complex. Science 307: 1098-1101, 2005

5. Manning BD and Cantley LC: AKT/PKB signaling: navigating downstream. Cell 129: 1261-1274, 2007.

6. Vazquez F and Sellers WR: The PTEN tumor suppressor protein: an antagonist of phosphoinositide 3-kinase signaling. Biochim Biophys Acta 1470: M21-M35, 2000.

7. Junttila MR, Li SP and Westermarck J: Phosphatase-mediated crosstalk between MAPK signaling pathways in the regulation of cell survival. FASEB J 22: 954-965, 2008.

8. Tanoue T and Nishida E: Docking interactions in the mitogenactivated protein kinase cascades. Pharmacol Ther 93: 193-202, 2002

9. Imajo M, Tsuchiya $\mathrm{Y}$ and Nishida E: Regulatory mechanisms and functions of MAP kinase signaling pathways. IUBMB Life 58: 312-317, 2006

10. Kant S, Schumacher S, Singh MK, Kispert A, Kotlyarov A and Gaestel M: Characterization of the atypical MAPK ERK4 and its activation of the MAPK-activated protein kinase MK5. J Biol Chem 281: 35511-35519, 2006

11. Depowski PL, Rosenthal SI and Ross JS: Loss of expression of the PTEN gene protein product is associated with poor outcome in breast cancer. Mod Pathol 14: 672-676, 2001.

12. Li J, Yen C, Liaw D, et al: PTEN, a putative protein tyrosine phosphatase gene mutated in human brain, breast, and prostate cancer. Science 275: 1943-1947, 1997.

13. Wu Z, Gioeli D, Conaway M, Weber MJ and Theodorescu D: Restoration of PTEN expression alters the sensitivity of prostate cancer cells to EGFR inhibitors. Prostate 68: 935-944, 2008.

14. Steelman LS, Navolanic PM, Sokolosky ML, et al: Suppression of PTEN function increases breast cancer chemotherapeutic drug resistance while conferring sensitivity to mTOR inhibitors. Oncogene 27: 4086-4095, 2008.

15. Nagata Y, Lan KH, Zhou X, et al: PTEN activation contributes to tumor inhibition by trastuzumab, and loss of PTEN predicts trastuzumab resistance in patients. Cancer Cell 6: 117-127, 2004.

16. Bianco R, Shin I, Ritter CA, et al: Loss of PTEN/MMAC1/TEP in EGF receptor-expressing tumor cells counteracts the antitumor action of EGFR tyrosine kinase inhibitors. Oncogene 22: 2812-2822, 2003.

17. Mason CS, Springer CJ, Cooper RG, Superti-Furga G, Marshall CJ and Marais R: Serine and tyrosine phosphorylations cooperate in Raf-1, but not B-Raf activation. EMBO J 18: 2137-2148, 1999.

18. Zimmermann S and Moelling K: Phosphorylation and regulation of Raf by Akt (protein kinase B). Science 286: 1741-1744, 1999.
19. Perren A, Weng LP, Boag AH, et al: Immunohistochemical evidence of loss of PTEN expression in primary ductal adenocarcinomas of the breast. Am J Pathol 155: 1253-1260, 1999.

20. Lu Y, Lin YZ, LaPushin R, et al: The PTEN/MMAC1/TEP tumor suppressor gene decreases cell growth and induces apoptosis and anoikis in breast cancer cells. Oncogene 18: 7034-7045, 1999.

21. Gu J, Tamura M and Yamada KM: Tumor suppressor PTEN inhibits integrin- and growth factor-mediated mitogen-activated protein (MAP) kinase signaling pathways. J Cell Biol 143: 1375-1383, 1998

22. Xie J, Qian J, Yang J, Wang S, Freeman ME III and Yi Q: Critical roles of Raf/MEK/ERK and PI3K/AKT signaling and inactivation of p38 MAP kinase in the differentiation and survival of monocyte-derived immature dendritic cells. Exp Hematol 33: 564-572, 2005.

23. Yoon S and Seger R: The extracellular signal-regulated kinase: multiple substrates regulate diverse cellular functions. Growth Factors 24: 21-44, 2006.

24. Kolch W: Coordinating ERK/MAPK signalling through scaffolds and inhibitors. Nat Rev Mol Cell Biol 6: 827-837, 2005.

25. Laprise P, Langlois MJ, Boucher MJ, Jobin C and Rivard N: Down-regulation of MEK/ERK signaling by E-cadherindependent PI3K/Akt pathway in differentiating intestinal epithelial cells. J Cell Physiol 199: 32-39, 2004.

26. Wen-Sheng W: Protein kinase $\mathrm{C}$ alpha trigger Ras and Rafindependent MEK/ERK activation for TPA-induced growth inhibition of human hepatoma cell HepG2. Cancer Lett 239: 27-35, 2006.

27. Albrecht DS, Clubbs EA, Ferruzzi M and Bomser JA: Epigallocatechin-3-gallate (EGCG) inhibits PC-3 prostate cancer cell proliferation via MEK-independent ERK1/2 activation. Chem Biol Interact 171: 89-95, 2008.

28. Grammer TC and Blenis J: Evidence for MEK-independent pathways regulating the prolonged activation of the ERK-MAP kinases. Oncogene 14: 1635-1642, 1997.

29. Kang-Park S, Lee YI and Lee YI: PTEN modulates insulin-like growth factor II (IGF-II)-mediated signaling; the protein phosphatase activity of PTEN down-regulates IGF-II expression in hepatoma cells. FEBS Lett 545: 203-208, 2003.

30. Hoeflich KP, O'Brien C, Boyd Z, et al: In vivo antitumor activity of MEK and phosphatidylinositol 3-kinase inhibitors in basal-like breast cancer models. Clin Cancer Res 15: 4649-4664, 2009.

31. Rexer BN, Ghosh R and Arteaga CL: Inhibition of PI3K and MEK: It is all about combinations and biomarkers. Clin Cancer Res 15: 4518-4520, 2009.

32. Schneider BP, Winer EP, Foulkes WD, et al: Triple-negative breast cancer: risk factors to potential targets. Clin Cancer Res 14: 8010-8018, 2008 